\title{
Identification of the Dynamic Properties of Al 5456 FSW Welds Using the Virtual Fields Method
}

\author{
G. Le Louëdec ${ }^{1,2} \cdot$ F. Pierron ${ }^{3}$ - M. A. Sutton ${ }^{2}$ C. Siviour ${ }^{4}$ A. P. Reynolds ${ }^{5}$
}

Received: 21 January 2015/Accepted: 16 March 2015/Published online: 24 March 2015

(C) Society for Experimental Mechanics, Inc 2015

\begin{abstract}
The present study focuses on the identification of the evolution of the dynamic elasto-plastic properties of Al 5456 FSW welds. An innovative method is proposed to make best use of the data collected with full-field measurements during dynamic experiments, and achieve identification of the mechanical properties of heterogeneous materials without requiring measurement of the load. Compressive specimens have been submitted to high strain-rate loading through a split Hopkinson pressure bar device while displacement fields were obtained using fullfield measurement techniques. Two sets of experiments have been performed using two different methods: the grid method and digital image correlation. Afterwards, the identification of the elastic and plastic properties of the material was carried out using the Virtual Fields Method. Finally, identification of the evolution of the yield stress throughout the weld has been achieved for strain-rates of the order of $10^{3} \mathrm{~s}^{-1}$.
\end{abstract}

\author{
F. Pierron \\ f.pierron@soton.ac.uk \\ G. Le Louëdec \\ g.lelouedec@gmail.com \\ M. A. Sutton \\ sutton@cec.sc.edu \\ C. Siviour \\ clive.siviour@eng.ox.ac.uk \\ A. P. Reynolds \\ reynolds@cec.sc.edu \\ 1 Laboratoire de Mécanique et Procédés de Fabrication, Arts et \\ Métiers ParisTech, Centre de Châlons-en-Champagne, Rue St \\ Dominique, BP 508, 51006 Châlons-en-Champagne Cedex, \\ France
}

Keywords Friction stir welding · Virtual fields method · Dynamic deformation - Digital image correlation · Grid method · Split Hopkinson pressure bar

\section{Introduction}

Since its invention in 1991, the friction Stir Welding (FSW) process [1] has allowed the use of large aluminium structures for a wide range of applications, thanks to the high resistance of the welds thus produced. In various fields, such as automotive and aeronautics, these welds hold an important place. Therefore, the evolution of the mechanical properties at different strain-rates is of interest; with the knowledge that, depending on the process, the welded material can undergo important structural changes, ranging from different grain size to a total recrystallisation. However, the high strain-rate mechanical properties used in numerical simulations are still estimates. Indeed, different

2 Department of Mechanical Engineering, Center for Mechanics, Material and NDE, University of South Carolina, 300 Main Street, Columbia, SC 29208, USA

3 Engineering and the Environment, University of Southampton, Highfield, Southampton SO17 1BJ, UK

4 Department of Engineering Science, University of Oxford, Parks Road, Oxford, UK

5 Department of Mechanical Engineering, Center for Friction Stir Welding, University of South Carolina, 300 Main Street, Columbia, SC 29208, USA 
issues arise when dealing with dynamic experiments. It is not easy to obtain accurate measurements of the strain, the load and the acceleration at strain-rates of the order of $10^{3} \mathrm{~s}^{-1}$ or more.

Several tests have been used over the last century to carry out experiments at high strain-rates [2]. The split Hopkinson pressure bar (SHPB) was developed based on the work of Hopkinson [3] and Kolsky [4]. This system allows the realization of experiments at strain-rates up to $10,000 \mathrm{~s}^{-1}$. Over the last decades, the SHPB and the tensile split Hopkinson bar [5] have become standards for the dynamic characterization of materials [6-14]. Starting with Hoge [15], the influence of the strain-rate on the mechanical properties of aluminium alloys, more specifically here, the tensile yield stress, has been investigated. For Al $6061 \mathrm{~T} 6$ and a strain-rate varying from 0.5 to $65 \mathrm{~s}^{-1}$, Hoge measured an increase in yield stress of approximately 28 $\%$. More recent work by Jenq et al. [8] showed the evolution of the stress-strain curve between compressive quasistatic and dynamic tests at strain-rates ranging from 1350 to $2520 \mathrm{~s}^{-1}$. In that work, increases in yield stress of $25 \%$ between the quasi-static test and the $1350 \mathrm{~s}^{-1}$ test and 60 $\%$ between the quasi-static and the $2520 \mathrm{~s}^{-1}$ test were measured. For Al 5083, Al 6061 and A356 alloys, it is also worth noting that Tucker [13] reported almost no evolution of the yield stress between tensile quasi-static and dynamic tests, also reaching similar conclusions for compression and shear. However, significant work hardening differences were recorded between tension and compression, with consistent increasing work hardening with strain-rate in compression.

To date, very few investigations have been conducted on the dynamic properties of welds. With SHPB experiments, it is possible to identify the average properties of a welded specimen [16-18]. However, there is no information about the local evolution of the dynamic properties within the weld. Due to the complex thermo-mechanical history of the welded material, the strain-rate dependence of the different areas of the weld could be quite different. Therefore, investigation of the evolution of the local properties of the material is of interest. Yokoyama et al. [19] proposed to carry out the identification of the dynamic local properties in a weld by cutting small specimens in the weld so as to consider each specimen as homogeneous. However, some issues remain due to the low spatial resolution and the assumption of the specimen homogeneity. This is also a very long and tedious process.

Developments in the field of digital ultra-high speed (UHS) cameras now allow the imaging of experiments at $10^{6}$ frames per second and above. The definition of 'ultrahigh speed imaging' is provided in [20]. Studies regarding the performance of high speed and ultra-high speed imaging systems have been reported in the past few years, e.g. [21, 22]. These technologies enable temporal resolutions on the order of a microsecond and below with good spatial resolution, making it possible to measure both fullfield strains and accelerations with excellent temporal and spatial resolutions; this is essential for the current study. These cameras still have important drawbacks however: high noise level, low number of images and very high cost. Recently, the advent of in-situ storage cameras like the Shimadzu HPV-X or the Specialized Imaging Kirana has given new impetus to using ultra-high speed imaging for full-field deformation measurements. Image quality has improved considerably, as evidenced in [23].

Finally, in dynamic testing, the key issue relates to external load measurement. Indeed, inertial effects in standard load cells ('ringing') prevent accurate loads to be measured. The alternative is to resort to an SHPB set-up using the bars as a very bulky and inconvenient load cell. This procedure works well but within a very restrictive set of assumptions: specimen quasi-static equilibrium (no transient stress waves, requiring a short specimen) and uniaxial loading, in particular. The need for more complex stress states to identify and fully validate robust constitutive models requires investigators to move away from such stringent assumptions if at all possible. The current study is exploring this idea for welds, based on the Virtual Fields Method (VFM).

The VFM was first introduced in the late 1980s in order to solve inverse problems in materials constitutive parameter identification with the aid of full-field measurements. Since then, it has been successfully applied to the identification of constitutive parameters for homogeneous materials in elasticity [24, 25], elasto-plasticity [26, 27], and visco-plasticity [28]. The method has also been used for heterogeneous materials (welds) in quasi-static loading and elasto-plastic material response [29]. Recent developments by Moulart et al. [30] introduced the application of the VFM to the identification of the dynamic elastic properties of composite materials. The main idea in this case is to use the acceleration field as a load cell, avoiding the need to measure an external load. Since then, it has also been used to identify the damage process of concrete materials [31], and to analyze the deformation of a beam in dynamic three-point bending [32]. More recently, spectacular improvements in image quality has led to unprecedented quality of identification, as evidenced in [23] for the elastic response of a quasi-isotropic laminate at strain-rates above $2000 \mathrm{~s}^{-1}$. However, until now, it is has never been attempted to identify an elasto-plastic model with this approach. Thus, the enclosed work breaks new ground by not only using acceleration fields instead of measured load data but also applying this approach in the more complex situation where heterogeneous plastic deformation is occurring in a weld. 
The aim of this study is to explore new ways to use the VFM for the identification of the dynamic heterogeneous elasto-plastic properties of Al $5456 \mathrm{FSW}$ welds. The nature of the paper is seminal in the way that it insists on the methodology and its potential. Many developments are still required to make this procedure a standard tool (including better UHS cameras, adapted test design etc.) but the authors feel that the current technique has great potential for future dynamic tests of materials. This study is part of a global long-term effort to design the next generation of high strain-rate tests based on rich full-field deformation information. The recent progress in UHS cameras reported above makes this contribution all the more timely, even if the results reported here are somewhat impaired by the fact that lower image quality cameras were used at the time that the experiments were performed.

\section{Specimens and Experiments}

The identification of the dynamic properties of the weld was performed based on experimental results from SHPB tests. It is worth noting however that the set-up of the SHPB test is used here, but the SHPB data reduction procedures are not used. Moreover, the first images were taken when the transient stress wave was present in the specimens and the accelerations were at their maximum, preventing any use of the standard SHPB analysis anyway. Two series of tests have been carried out for this work: one at the University of Oxford on welded and base material specimens where the grid method (or 'sampling moiré') [33-39] was used, and a second one at the University of South Carolina on welded specimens only where digital image correlation [40] was used.

\section{Specimens}

Generally, cylindrical specimens are used in the SHPB setup to ensure a homogeneous propagation of the wave. However, in this case, 2D imaging was performed during the experiment. Therefore, flat surfaces were machined on both sides of the specimens (Fig. 1) in order to comply with 2D-DIC and grid method requirements. The specimen was designed so as to avoid any compressive buckling during the early part of the test when measurements were collected. Indeed, for this kind of specimen, buckling will occur for an axial stress of about $420 \mathrm{MPa}$, when the expected dynamic yield stress for the base material (50\% higher than the quasi-static value [13]) is $380 \mathrm{MPa}$. Therefore, information on the elasto-plastic behaviour will be available before any buckling occurs.

\section{SHPB Tests Using the Grid Method}

These tests were performed on a SHPB set-up at the University of Oxford. Five tests were performed with the grid method, three on base material specimens and two on welded specimens.

\section{Experiment}

Before performing the experiments, cross-line grids have been transferred onto the surface of interest of the different specimens. The grids were printed on a $0.18 \mathrm{~mm}$ thick polyester film, with a period of $150 \mu \mathrm{m}$. The grid transfer was performed using the method proposed by Piro and Grédiac [41]. The imaging field of view was $24.5 \mathrm{~mm}$ along the $X_{2}$-direction (starting on the left hand side of the specimen) and $10 \mathrm{~mm}$ along the $X_{1}$-direction which is the

Fig. 1 Schematic of a welded specimen with the retreating side on the right and the impact side on the left. The red lines represent the position of the nugget (centre) and the limit of the welded zone (Color figure online)
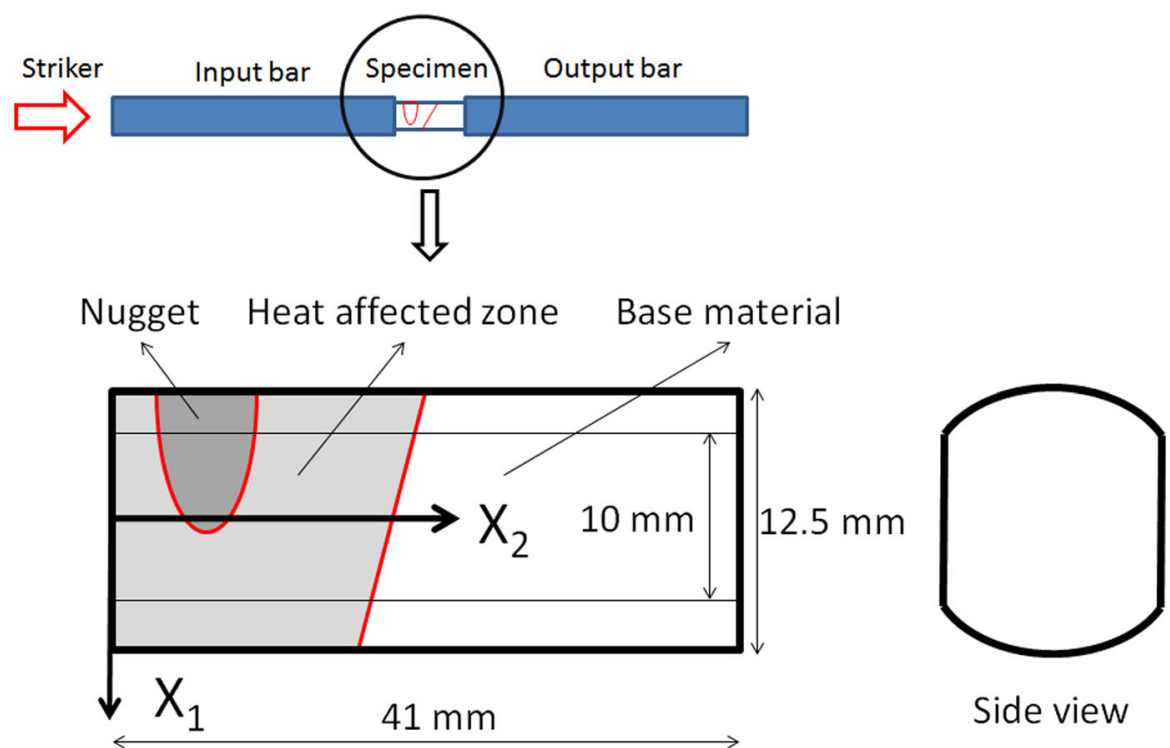
width of the flattened side of the specimen, see Fig. 1. The camera used here is a SIM 16 camera with a $50 \mathrm{~mm}$ lens. This camera possesses 16 CCD sensors and a beam splitter spreading the light through the 16 channels, enabling extremely fast imaging as the limiting factor is electronic gating. However, the downside of this technology concerns the use of light amplifiers (ICCD sensors) causing issues in the imaging, as will be demonstrated later on in this article and illustrated in previous studies [21,22]. Some details concerning the camera and lens are reported in Table 1. The camera was positioned facing the grid with the lens axis normal to the observed surface, with the specimen approximately $20 \mathrm{~cm}$ from the lens. The specimen was illuminated using two flash lights triggered from a strain gauge bonded onto the incident bar. In dynamics, wave propagation is much faster than any rigid body movements. Therefore, it is favourable for 2D imaging with a camera positioned close to the specimen, as the issue of parasitic strain coming from out-of-plane displacements can largely be ignored since the strain wave has passed before the out of plane motions occur. The quantification of the noise level was performed by measuring the displacement between two sets of images of the stationary specimen and calculating the standard deviation of the resulting displacement and strain fields.

Both input and output bars were $500 \mathrm{~mm}$ long, $15 \mathrm{~mm}$ in diameter and made from steel. The impactor speed was up to $18 \mathrm{~m} \mathrm{~s}^{-1}$. The strain-rate fields obtained by finite difference differentiation of the strain fields showed maximum local strain-rates of respectively 1300 and $1000 \mathrm{~s}^{-1}$ for welded and homogeneous specimens. It should be noted that the strain-rate maps are heterogeneous in space and variable in time. In particular, at the onset of plasticity, there is a sharp local increase of the strain-rate, as was also evidenced in [28]. In the standard SHPB approaches, this is ignored and only an average strain-rate is considered. Ideally, the heterogeneous strain-rate maps should be used to enrich the identification of the strain rate dependence, as was performed in [28]. This was not done here as the quality of the data does not currently allow for it, but this is a clear track to follow in the future to improve the procedure. The acquisition and lighting systems were triggered by a strain gauge bounded onto the incident bar. The images were taken with an interframe time of $5 \mu \mathrm{s}$, and a shutter speed of $1 \mu \mathrm{s}$. A total of 16 images were taken during each test. Indeed, the technology of the camera is based on the use of a beam splitter and 16 sensors. Therefore, each image was taken from a different sensor. As a result, there was a difference in light intensity between the different images, and it was not possible to accurately measure the displacement fields between images taken from different sensors. To address this issue, the displacement fields were computed between two images taken with the same sensor: one static reference image and the actual dynamic one. The displacement computation is based on the phase shift between the reference and deformed images [33]. In this study, a windowed discrete Fourier transform (WDFT) algorithm was used [34, 35, 42, 43]. It calculates the discrete Fourier transform of the intensity over a set of pixels over a triangular windowed
Table 1 SHPB imaging parameters with SIM 16 camera

\begin{tabular}{ll}
\hline Camera & SIM 16 \\
Sensor size & $1360 \times 1024$ pixel $^{2}$ \\
Field of view & $24.5 \times 10 \mathrm{~mm}^{2}$ \\
Interframe & $5 \mu \mathrm{s}$ \\
Shutter speed & $1 \mu \mathrm{s}$ \\
Total number of images & 16 \\
Technique used & Grid method \\
$\quad$ Period size & $150 \mu \mathrm{m}$ \\
Pixels per period & 9 \\
Displacement & \\
Smoothing method & Least square convolution \\
Smoothing window & $31 \times 31$ measurement points \\
Resolution & 0.048 pixels $(0.8 \mu \mathrm{m})$ \\
Strain & \\
Differentiation method & Finite difference \\
Resolution & $313 \mu$ strain \\
Acceleration & \\
Differentiation method & Finite difference from smoothed displacements \\
Resolution & $66,000 \mathrm{~m} \mathrm{~s}{ }^{-2}$ \\
\hline
\end{tabular}


Table 2 SHPB imaging parameters with DRS IMACON 200 camera

\begin{tabular}{ll}
\hline Camera & IMACON 200 \\
Sensor size & $1340 \times 1024$ pixel $^{2}$ \\
Field of view & $24.5 \times 10 \mathrm{~mm}^{2}$ \\
Interframe & $4 \mu \mathrm{s}$ \\
Shutter speed & $\sim 0.4 \mu \mathrm{s}$ \\
Total number of images & 16 \\
Technique used & DIC \\
Speckle pattern & Rub on transfer decal \\
Subset & 55 \\
Shift & 20 \\
Displacement & \\
Smoothing method & Least square convolution \\
Smoothing window & $31 \times 31$ measurement points \\
Resolution & 0.07 pixels $(1.28 \mu \mathrm{m})$ \\
Strain & \\
Differentiation method & analytical \\
Resolution & $484 \mu$ strain \\
Acceleration & \\
Differentiation method & Finite difference from smoothed displacements \\
Resolution & $45,000 \mathrm{~m}$ s \\
\hline
\end{tabular}

kernel. However, the measured phase maps consist of values between $-\pi$ and $\pi$. Therefore, it is not possible to measure a displacement associated to a phase shift that exceeds $\pi$. In this case, it is necessary to unwrap the phase map in order to obtain the actual value of the displacement. Extensive work has been done in the past to address this problem [44-46]. The algorithm used in this study is presented in [47].

\section{Smoothing, Acceleration and Strain Computation}

In order to reduce the effect of measurement noise, displacement fields were smoothed using an iterative least square convolution method [48]. The smoothing was performed over a $31 \times 31$ pixels window using a second order polynomial function. Then, the strain fields were computed from the displacement fields by finite difference. Velocity and acceleration fields were calculated from the smoothed displacement field using a centred temporal finite difference scheme. This precluded reliable acceleration maps to be obtained for the two first and last images. Therefore, acceleration fields were only available for 12 steps of the experiment, when 16 steps were available for the strain and displacement fields. By recording two sets of images for the stationary specimen prior to testing, it is possible to compute the standard deviation of the resulting displacement, strain and acceleration maps. This provides an estimate of the 'resolution' as reported in Table 1 together with smoothing details.

\section{SHPB Tests Using Digital Image Correlation}

This test was carried out on a welded specimen with digital image correlation on an SHPB set-up at the University of South Carolina.

\section{Experiment}

Before performing the experiment, the specimen was coated with a thin layer of white paint and a black random speckle pattern was transferred on it using rub on transfer decal paper. This method was preferred to the use of paint and airbrush to obtain a highly contrasted speckle pattern. The reasoning behind this choice will be developed in the next section. The field of view of the camera was $24.5 \mathrm{~mm}$ along the $X_{2}$-direction (starting on the left side of the specimen) and $10 \mathrm{~mm}$ along the $X_{1}$-direction which is the width of the flattened side of the specimen. The camera used here was a DRS IMACON 200 with a $200 \mathrm{~mm}$ lens (Table 2). The camera was positioned facing the specimen with the lens axis normal to the observed surface. The specimen was lit by two flash lights. The quantification of the noise level was performed by measuring the displacement between two sets of static images and calculating the standard deviation of the resulting displacement and strain fields.

Both input and output bars were $2388 \mathrm{~mm}$ long, 25.4 $\mathrm{mm}$ in diameter and made from steel. The $483 \mathrm{~mm}$ long steel impactor speed was $24 \mathrm{~m} \mathrm{~s}^{-1}$. The strain-rate fields measured by finite difference of the strain fields showed a 
(a)
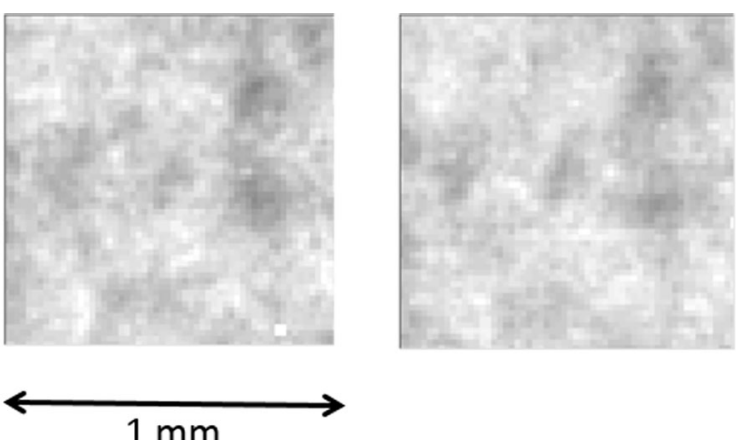

(b)
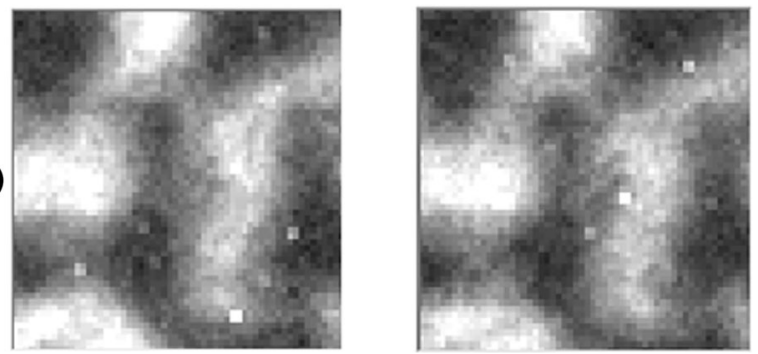

Fig. 2 Same subset on 2 different static images taken with the Imacon 200 with a spray paint speckle pattern and $\mathbf{b}$ rub on transfer decal speckle pattern. Both set of images were taken on the same setup and under the same lighting condition and grey level scale

maximum local strain-rate of $1600 \mathrm{~s}^{-1}$. The acquisition system was triggered by a piezo-electric sensor set on the incident bar. The images were taken with an interframe time of $4 \mu \mathrm{s}$, and a shutter speed of $0.4 \mu \mathrm{s}$. The DRS IMACON 200 uses the same type of technology as the SIM 16 , therefore displacement fields were computed between a static reference image and the actual dynamic image from each sensor. Moreover, in order to reduce the influence of the difference of contrast between the different sensors, flat-field correction has been performed on the images [22]. All images were processed using the 2D-DIC software VIC-2D [49].

\section{Noise Issues}

Due to the technology of the DRS IMACON 200, the noise level remains an issue. In fact, the camera tends to smooth out the grey levels on the raw images (Fig. 2). This is caused by pixel to pixel photon "leakage" due to the light amplifiers. It is worth noticing that the same issue arises with the SIM 16 camera, however, the phenomenon was less marked probably because of the lower grain size in the phosphorous screens used in the light amplifiers. As a consequence, it was chosen to realise the speckle pattern by using a rub on transfer decal paper instead of spray paint. Thanks to the highly contrasted speckle pattern, it has been possible to reduce the effects of the high noise level (Fig. 3). Despite this improvement, the noise level remains (a)

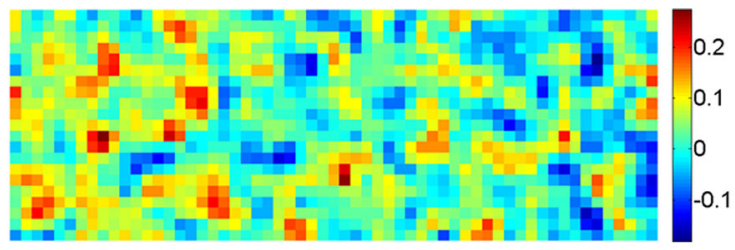

(b)

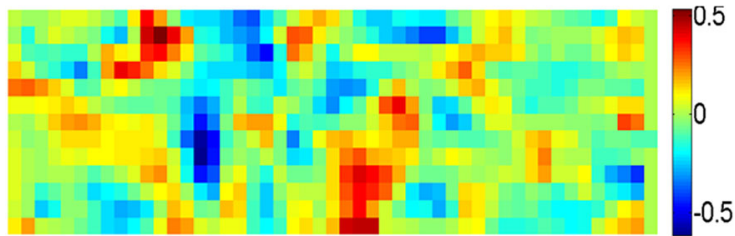

Fig. 3 a Raw displacement field in pixels between two static images for a rub on transfer decal speckle pattern. b Raw displacement field in pixels between two static images for a spray paint speckle pattern

significant. This matter was investigated by Tiwari et al. [22] who recommended the use of unusually large subsets at the cost of spatial resolution. Therefore, to ensure accurate measurement of the displacement fields, a subset of 55 pixels was used. However, a second issue arose. Even with a large subset, the noise presented a high spatial correlation (Fig. 3). This will remain a problem as it will make smoothing less efficient. Nevertheless, the spatial heterogeneities of the mechanical fields are limited, which is the reason why the current limitations can be overcome and quantitative data produced.

\section{Smoothing, Acceleration and Strain Computation}

The strain fields were computed by analytical differentiation after least square quadratic fit over a $5 \times 5$ window of the displacement fields by the VIC-2D software [49]. Then the strain fields were smoothed using an iterative least square convolution method [48] over a $31 \times 31$ pixels window using a second order polynomial function. The calculation of the acceleration fields was performed with the method used for the computation of the measurement from grid method tests. The baseline information on the measurements can be found in Table 2 .

\section{Results}

The evolution of the axial strain and acceleration fields are presented in Figs. 4, 5, 6, 7, 8, and 9. Foremost, it is important to note that there is a time shift between the two set-ups. Indeed, the triggering was not performed in the same manner. As a result, the earliest stages of the mechanical wave do not appear on the acceleration fields from the set-up using the grid method. On the first acceleration field, the mechanical wave is already halfway through the specimen which corresponds to the third field $(16 \mu \mathrm{s})$ measured with DIC. However, in Figs. 5, 7 and 9, the 


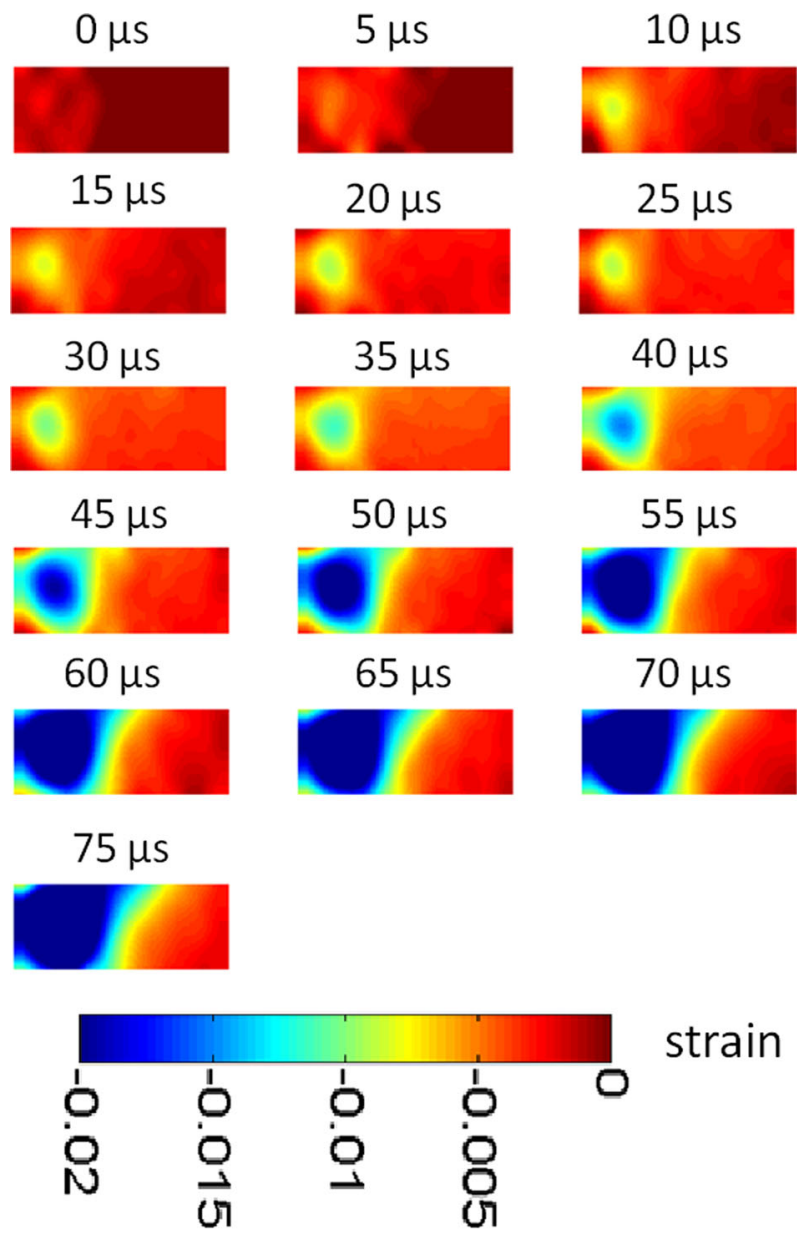

Fig. 4 Measurement with the grid method of the evolution of the axial strain field over time for a base material specimen

impact wave is clearly visible at the early stages of the experiment (acceleration $>0$ ), which is followed by a reflected wave (acceleration $<0$ ) and a second reflection of the wave (acceleration $>0$ ) of lower magnitude. It should be noted that the elastic strains caused by the elastic wave cannot be seen on the strain maps as they are hidden in the large plastic strains present in the specimen. It is also worth noting that, for both welded and homogeneous specimens, there is a strong localisation of strain on the impact side. For base material specimens, this is mostly due to a non-uniform contact between the impacting bar and the specimen, while the gradient of mechanical properties is responsible for it in the case of welded specimens. It should be noted however that this is not a problem for the analysis performed in this paper as the inverse identification naturally folds this in.

Concerning the measurements with the grid method, the lower impact velocity could affect the identification of the mechanical properties of the material. Indeed, it results in lower values in the acceleration and strain fields and

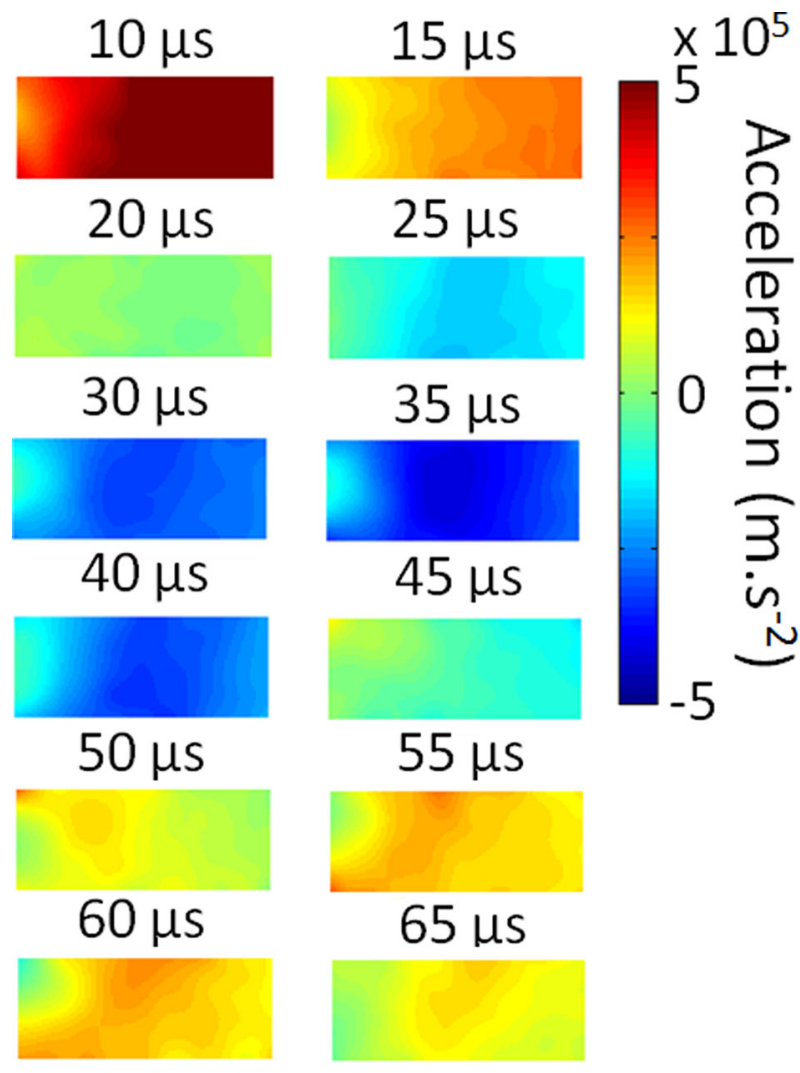

Fig. 5 Measurement with the grid method of the evolution of the axial acceleration field (in $\mathrm{m} \mathrm{s}^{-2}$ ) over time for a base material specimen

therefore, could hinder the identification process due to larger noise to signal ratio. One can notice that the average strain on the right hand side of the specimen barely reaches the estimated base material yield strain $(\simeq 0.005)$. Therefore, it could affect the identified plastic parameters. This problem does not occur in the measurements realised with DIC due to the higher impact velocity and average strain over the specimen.

\section{Virtual Fields Method}

The virtual fields method is based on the principle of virtual work, which is written, in absence of volume forces, as (1). The convention of summation over repeated indices is used here.

$-\iiint_{V} \sigma_{i j} \epsilon_{i j}^{*} d V+\iint_{S_{V}} T_{i} u_{i}^{*} d S=\iiint_{V} \rho a_{i} u_{i}^{*} d V$

$(i, j)=(1,2,3)$ 


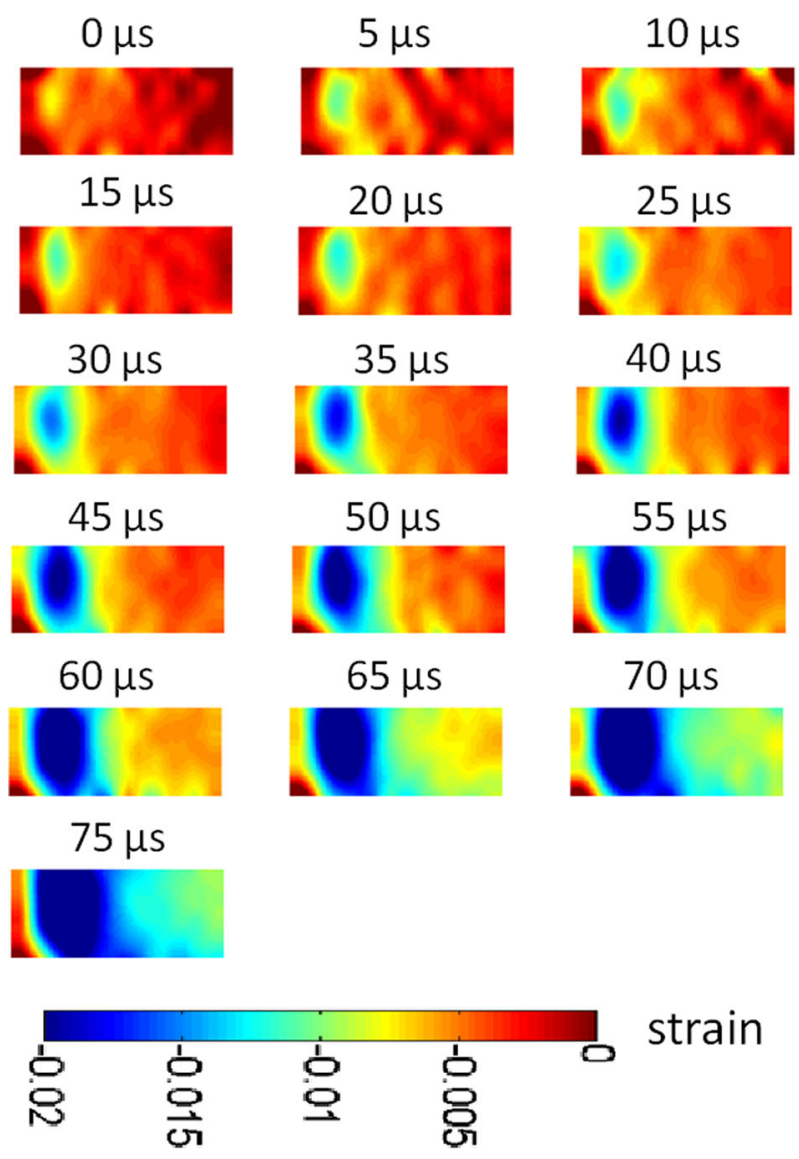

Fig. 6 Measurement with the grid method of the evolution of the axial strain field over time for a welded specimen

$T_{i}=\sigma_{i j} n_{j}$ over $S_{V}$

with: $\sigma_{i j}$ the stress tensor, $\rho$ the density of the material, $a_{i}$ the acceleration vector, $V$ the volume where the equilibrium is written, $u_{i}^{*}$ the virtual displacement field, $\epsilon_{i j}^{*}$ the virtual strain tensor deriving from $u_{i}^{*}, S_{V}$ the boundary surface of $\mathrm{V}, T_{i}$ the imposed traction vector over the boundary $S_{V}$. In the case of dynamic experiments, load measurement is an issue. Therefore, in order to cancel out the contribution of the load in the principle of virtual work (PVW), a specific virtual field is used that must comply with the specification described in (4).

$\iint_{S_{V}} T_{i} u_{i}^{*} d S=0$

Then, by replacing (4) into (1) a new formulation of the PVW for dynamic loading is obtained (5).

$-\iiint_{V} \sigma_{i j} \epsilon_{i j}^{*} d V=\iiint_{V} \rho a_{i} u_{i}^{*} d V$

Therefore a relationship is obtained between the stress field and the acceleration field. Then, with the assumption that

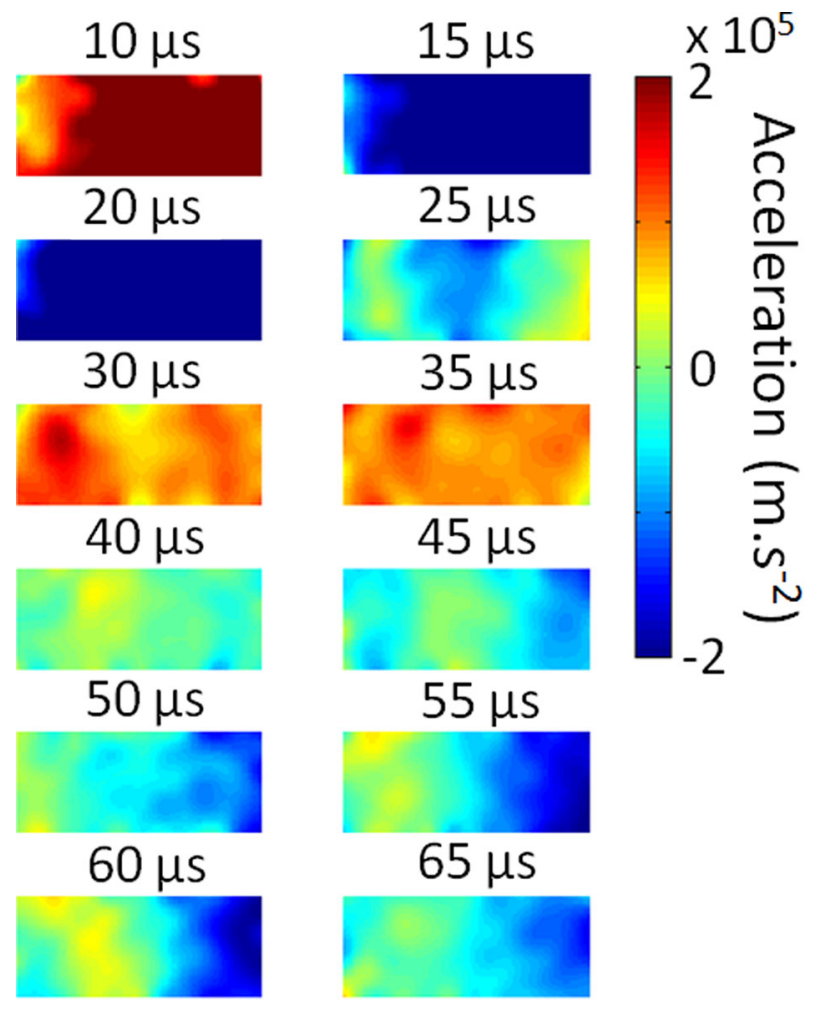

Fig. 7 Measurement with the grid method of the evolution of the axial acceleration field (in $\mathrm{m} \mathrm{s}^{-2}$ ) over time for a welded specimen

the mechanical fields are uniform through the thickness and that the virtual fields are selected so that they do not depend on the through-thickness coordinates, (5) is developed into (6).

$-\iint_{S} \sigma_{i j} \epsilon_{i j}^{*} d S=\iint_{S} \rho a_{i} u_{i}^{*} d S$

It is interesting to note that this equation is valid on any surface of the specimen. Therefore, it is possible to carry out a local identification of the mechanical parameters without any consideration for what happens outside of this zone.

\section{Virtual Fields in Elasticity}

The identification of Young's modulus and Poisson's ratio was performed on the whole specimen which was considered as a homogeneous material. In order to perform this identification during the elastic steps of the test, two virtual fields are necessary and both of them have to comply with (4). The virtual fields defined in (7) and (8) have been used.

$$
\begin{aligned}
& \left\{\begin{array}{l}
u_{1}^{*(1)}=0 \\
u_{2}^{*(1)}=x_{2}\left(x_{2}-L\right)
\end{array}\right. \\
& \left\{\begin{array}{l}
u_{1}^{*(2)}=0 \\
u_{2}^{*(2)}=x_{2}^{2}\left(x_{2}-L\right)
\end{array}\right.
\end{aligned}
$$




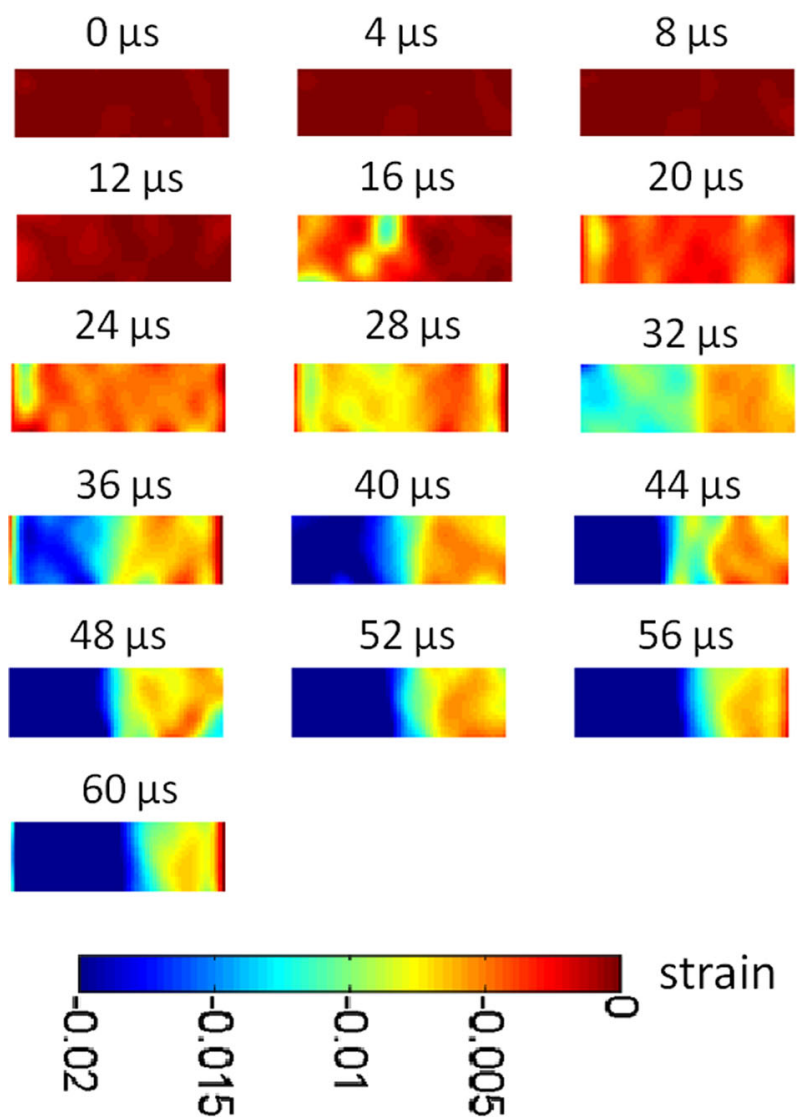

Fig. 8 Measurement with DIC of the evolution of the axial strain field over time for a welded specimen

where $L$ is the length of the identification area. By incorporating (7) and (8) into (6), the following system is obtained, assuming plane stress, linear isotropic elasticity and homogeneous elastic properties, as it has been shown in Sutton et al. [29] for quasi-static properties.

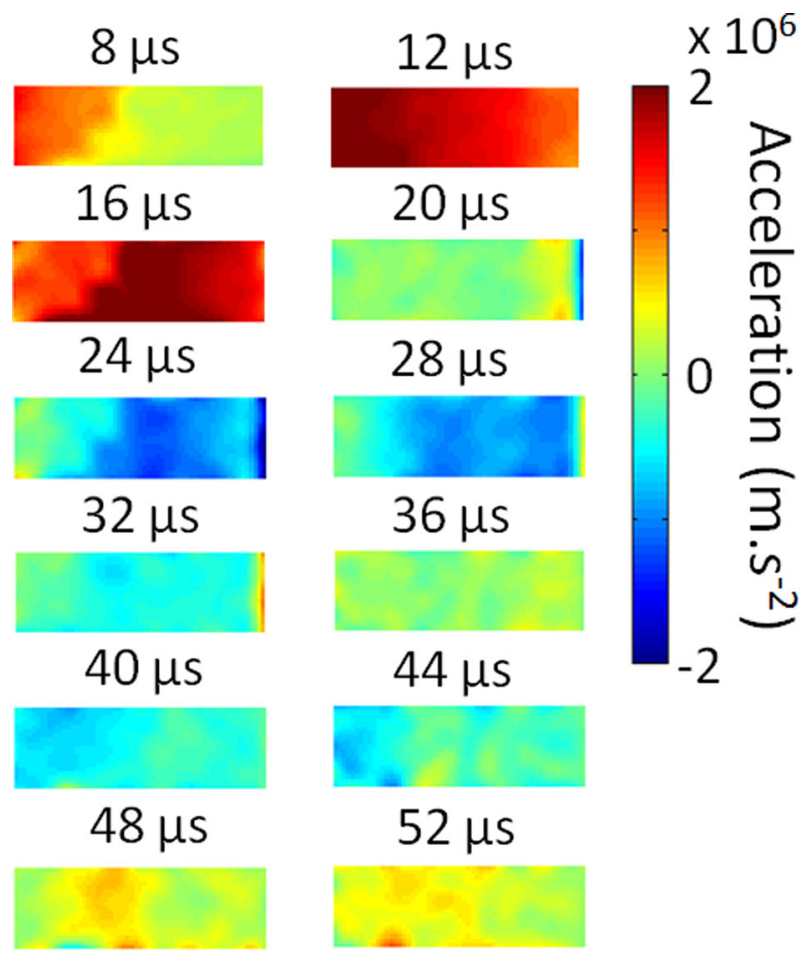

Fig. 9 Measurement with DIC of the evolution of the axial acceleration field (in $\mathrm{m} \mathrm{s}^{-2}$ ) over time for a welded specimen

frequency content of the mechanical fields and the spatial resolution of the measurements.

$\iint_{S} \varepsilon_{i j} d S \simeq \frac{L w}{N} \sum_{k=1}^{N} \varepsilon_{i j}^{(k)}=L w \bar{\varepsilon}_{i j}$

where the overline indicates spatial averaging over the area under consideration. This leads to a new formulation of (9) reported in (11).

$$
\left\{\begin{array}{c}
-\frac{E}{1-v^{2}} \iint_{S}\left(2 x_{2}-L\right) \varepsilon_{22} d S-\frac{v E}{1-v^{2}} \iint_{S}\left(2 x_{2}-L\right) \varepsilon_{11} d S \\
=\rho \iint_{S} x_{2}\left(x_{2}-L\right) a_{2} d S \\
-\frac{E}{1-v^{2}} \iint_{S}\left(3 x_{2}^{2}-2 x_{2} L\right) \varepsilon_{22} d S-\frac{v E}{1-v^{2}} \iint_{S}\left(3 x_{2}^{2}-2 x_{2} L\right) \varepsilon_{11} d S \\
=\rho \iint_{S} x_{2}^{2}\left(x_{2}-L\right) a_{2} d S
\end{array}\right.
$$

Full-field measurements are available over the surface of the specimen during the experiment. In order to carry out the identification of the elastic parameters, the integrals over the surface are approximated by discrete sums (see for instance (10)) with $w$ the width of the specimen, $N$ the number of measurement points over the area, and the bar indicating spatial averaging over the field of view. The quality of this approximation is dependent on the spatial

$$
\left\{\begin{array}{l}
-\frac{E}{1-v^{2}} \overline{\left(2 x_{2}-L\right) \varepsilon_{22}}-\frac{v E}{1-v^{2}} \overline{\left(2 x_{2}-L\right) \varepsilon_{11}}=\rho \bar{x}_{2}\left(x_{2}-L\right) a_{2} \\
-\frac{E}{1-v^{2}} \overline{\left(3 x_{2}^{2}-2 x_{2} L\right) \varepsilon_{22}}-\frac{v E}{1-v^{2}} \overline{\left(3 x_{2}^{2}-2 x_{2} L\right) \varepsilon_{11}}=\rho \bar{x}_{2}^{2}\left(x_{2}-L\right) a_{2}
\end{array}\right.
$$

Then (11) is first solved for $E /\left(1-v^{2}\right)$ and $v E /\left(1-v^{2}\right)$ by inversion of the linear system. Then, $E$ and $v$ are calculated from these quantities. 


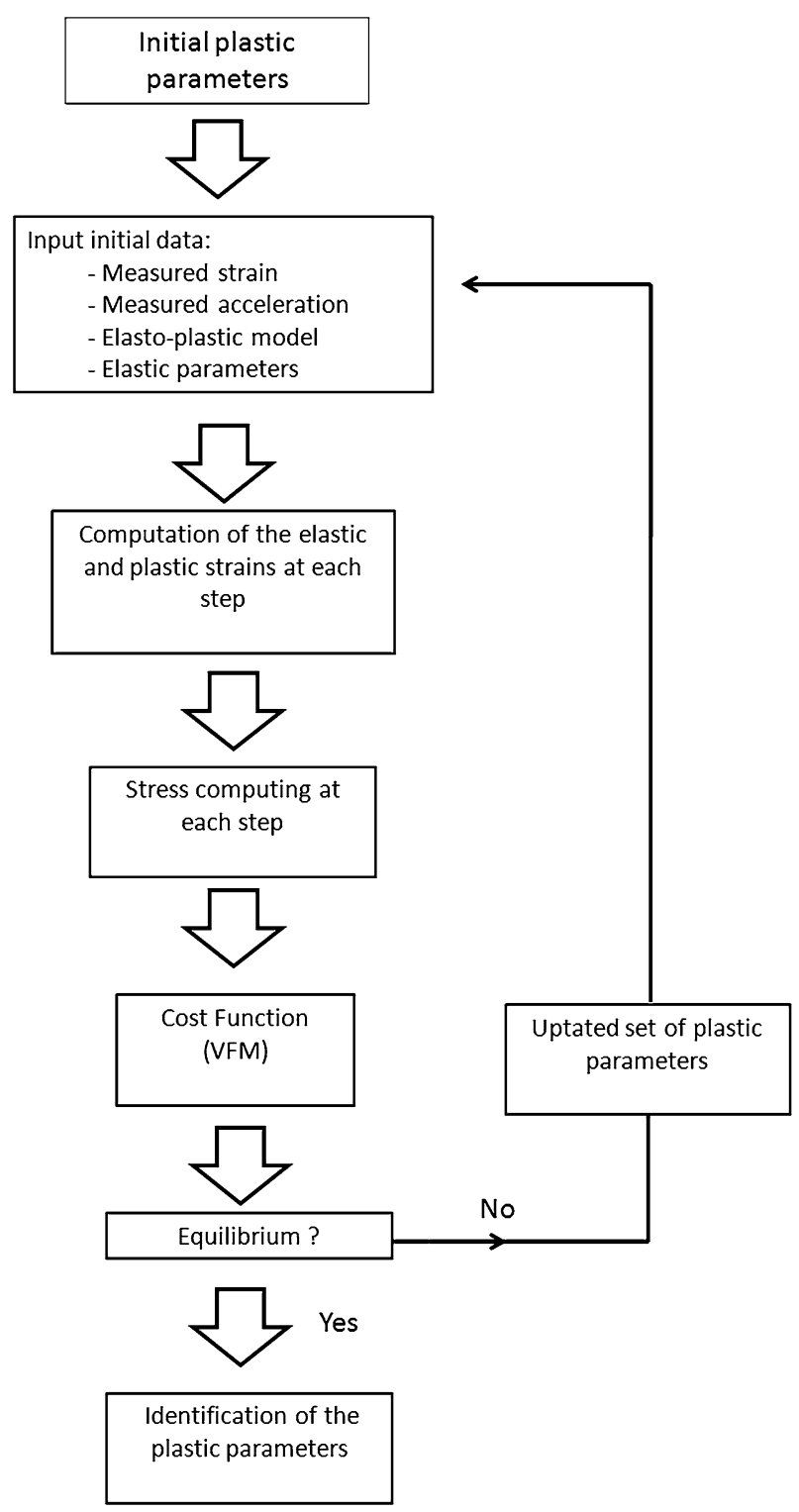

Fig. 10 Flowchart of the identification of plastic parameters using the VFM

\section{Virtual Fields in Homogeneous Plasticity}

The elasto-plastic model used in this study is very simple. It assumes Von-Mises yield function with associated plasticity and isotropic hardening. As a first attempt to keep things simple, a linear hardening model is selected. As a consequence, the model only involves the yield stress $\left(\sigma_{y}\right)$ and the hardening modulus $(H)(12)$.

$$
\sigma(t)=f\left(\varepsilon, E, v, \sigma_{y}, H, t\right)
$$

Due to the non-linearity of the stress-strain relationship in plasticity, it is not possible to extract the mechanical parameters from the first integral, and carry out the identification as in elasticity. This problem has been solved by
Grédiac and Pierron [27]. The identification has been carried out by constructing a cost function dependent on the plastic parameters (13). This function is the sum of the quadratic difference of the two terms in Eq. (6) over time.

$$
\Phi\left(\sigma_{y}, H\right)=\sum_{t=t_{0}}^{t_{f}}\left[\iint_{S} \sigma_{i j}\left(\varepsilon, E, v, \sigma_{y}, H, t\right) \varepsilon_{i j}^{*} d S+\iint_{S} \rho a_{i} u_{i}^{*} d S\right]^{2}
$$

In order to carry out the identification of the plastic parameters, the integrals over the surface are approximated by discrete sums as it has been done in Eq. (11). It leads to a new formulation of Eq. (13) shown in Eq. (14). The plastic parameters are then identified by minimization of the following cost function (Fig. 10).

$$
\Phi\left(\sigma_{y}, H\right)=\sum_{t=t_{0}}^{t_{f}}\left[\overline{\sigma_{i j}\left(\varepsilon, E, v, \sigma_{y}, H, t\right) \varepsilon_{i j}^{*}}+\rho \overline{a_{i} u_{i}^{*}}\right]^{2}
$$

Moreover, the stress-strain relationship being non-linear, a single virtual field is generally sufficient to perform identification when the number of parameters is low, which is the case here [27]. In order to calculate the value of $\Phi\left(\sigma_{y}, H\right)$, the stress field is computed at each step of the experiment using the method proposed by Sutton et al. [50]. This is an iterative method based on the radial return. The minimisation of the cost function is based on the Nelder-Mead simplex method [51].

\section{Virtual Fields in Plasticity for Heterogeneous Materials}

As opposed to the situation where a homogeneous material is studied, as in [26, 52], the elasto-plastic parameters within the weld depend on the space variables. Different strategies have been devised in the past to parameterize this variation: identify distinct zones based on strain localization (or microstructure), as in [29] or consider the properties constant over a certain transverse slice of the weld, as in [53]. This is the approach used here. For each of the nine shaded slices on Fig. 11, the following virtual field is used: $\left\{\begin{array}{l}u_{1}^{*(1)}=0 \\ u_{2}^{*(1)}=x_{2}\left(x_{2}-L\right)\end{array}\right.$

It is worth noting that the $\left(X_{1}, X_{2}\right)$ reference frame is a local frame linked to each individual slice. On Fig. 11, it is given for the first slice. By replacing (15) into (14), the following formulation of the cost function is obtained for each slice (numbered $(i)$ ).

$$
\begin{aligned}
\Phi^{(i)}\left(\sigma_{y}^{(i)}, H^{(i)}\right)= & \sum_{t=t_{0}}^{t_{f}} \overline{\left[\left(2 x_{2}-L\right) \sigma_{22}\left(\varepsilon, E, v, \sigma_{y}^{(i)}, H^{(i)}, t\right)\right.}{ }^{(i)} \\
& +{\overline{x_{2}\left(x_{2}-L\right) \rho a_{2}(t)}}^{2(i)}
\end{aligned}
$$


Here, only one virtual field has been used. Experience has shown that this was generally sufficient for a predominantly unidirectional stress state when considering isotropic yield surfaces. However, in spite of the work reported in [52], the optimization of the choice of virtual fields for non-linear constitutive models is still very much an open problem. It must also be understood that the thickness of the slices represents a compromise between a thin slice for better spatial resolution and a thick slice for lower influence of noise thanks to the spatial averages in Eq. 16. Here, the slices are much thicker than in [53] because the measurements are of much lower quality due to the noise levels present in the images obtained with the two ultra-high speed cameras.

\section{Results}

\section{Elastic Parameters}

The noise level in the measured displacement data remains the main issue when carrying out the identification of the mechanical parameters under high strain-rate. This point becomes more critical for the elastic parameters due to the high noise to signal ratio. Because of this issue, it was not possible to retrieve the elastic parameters for the test using DIC. For the experiments performed with the SIM16 camera, the identification was completed using only the data from the elastic steps of the tests. The results are presented in Table 3. The quasi-static reference values (as given by the supplier) of these parameters have been added in order to give a reference for the results obtained. These results still exhibit relatively large dispersion. Nevertheless, the results are promising since the accuracy of the extracted parameters will improve with the quality of images, which is already happening with the new generation of UHS cameras based on in-situ image storage, allowing unprecedented image quality and ease of use, as evidenced in [23]. In fact, the values obtained for the welded specimen are much better because of the better image quality for this particular test.

Table 3 Elastic parameters identified by the VFM

\section{Plastic Parameters}

The reference value of Young's modulus and Poisson's ratio were used for the identification of the plastic parameters. The identification was carried out using the images from the plastic steps of the tests. Moreover, due to the fact that all areas of the weld do not yield at the same time and do not undergo the same amount of strain, the identification has been carried out using different numbers of images for each slice. Indeed, with the slices on the

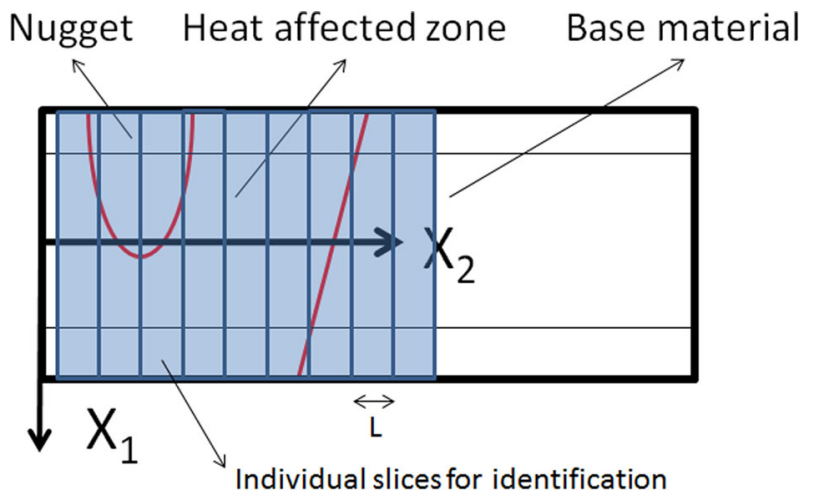

Fig. 11 Schematic of the different slices of the specimen

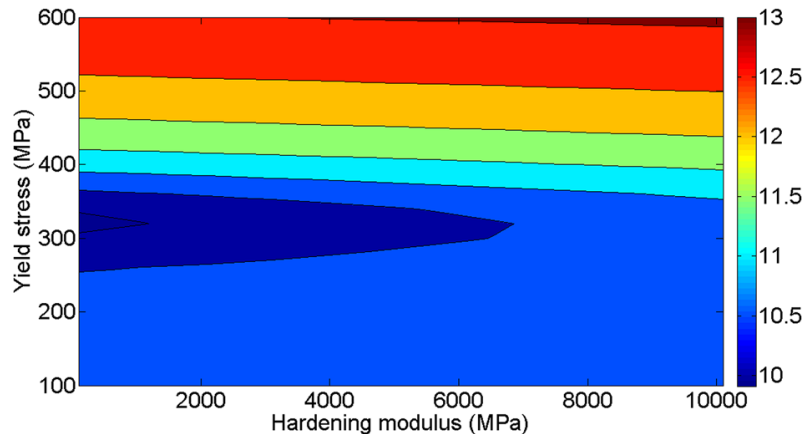

Fig. 12 Representation of the logarithm of the VFM cost function for a slice at the centre of a base material specimen (grid method, specimen divided up in six slices)

\begin{tabular}{llll}
\hline & Reference & Grid: base material & Grid: welded specimen \\
\hline Young's modulus (GPa) & & & \\
$\quad$ First test & 70 & 62 & 68 \\
Second test & 70 & 32 & 72 \\
Third test & & & \\
Poisson's ratio & 0.33 & 0.1 & 0.31 \\
First test & 0.33 & 0.1 & 0.37 \\
Second test & 0.33 & 0.7 & \\
Third test & & & \\
\hline
\end{tabular}


Table 4 Plastic parameters identified by the VFM for base material specimens using the grid method

\begin{tabular}{lllll}
\hline & Reference & $\begin{array}{l}\text { Base } \\
\text { material 1 }\end{array}$ & $\begin{array}{l}\text { Base } \\
\text { material 2 }\end{array}$ & $\begin{array}{l}\text { Base } \\
\text { material 3 }\end{array}$ \\
\hline $\begin{array}{c}\text { Yield stress } \\
(\mathrm{MPa})\end{array}$ & 255 & 368 & 376 & 402 \\
\hline
\end{tabular}

impact side of the specimen, the strain level is more important and yield occurs earlier. Therefore, 5-8 images were used to perform the identification, depending on the slice. Knowing that the identification makes use of a minimisation process, the starting values of the algorithm could have an impact on the identified parameters. The evolution of the cost function with the plastic parameters is represented in Fig. 12, for a slice in the centre of a base material specimen.

It is important to note that if the cost function admits a clear minimum value for variations of the yield stress, it is almost insensitive to the variation of the hardening modulus. This problem can be addressed by increasing the number of images in the cost function. Unfortunately, the number of available images for the dynamic tests was very limited. As a result, it has not been possible to carry out the identification of the hardening modulus, and only the yield stress has been identified. Figure 12 shows that the cost function admits a clear minimum over a range of reasonable values for the yield stress. The identification has been performed using a starting point of $200 \mathrm{MPa}$. For the base material specimens, the identification has been carried out over the whole field of view. The results are presented in Table 4. The quasi-static value for the yield stress has been added as a reference [54]. A steady identification (i.e. convergence was achieved) of the yield stress has been obtained on these specimens, with an average value of 382 $\mathrm{MPa}$. These values are about $50 \%$ higher than the quasistatic reference and are consistent with the results obtained by Tucker [13]. In practice, the choice of the slice width (or the choice of the total number of slices within the field of view) will determine how finely the spatial yield stress distribution can be described within the weld. Indeed, a sharp change of yield stress within a slice will be smoothed out by the fact that a constant yield stress is identified over this slice. Considering this, one would want to go for thinner slices but then, the spatial averages in Eq. 16 will be less efficient at filtering out measurement noise so a compromise has to be found. In order to investigate this issue and help select a typical slice width, the base material specimens have been divided up in slices and the identification performed as it will be on the weld specimens. Ideally, the yield stress values obtained in each slice should be identical but because of measurement noise, they are

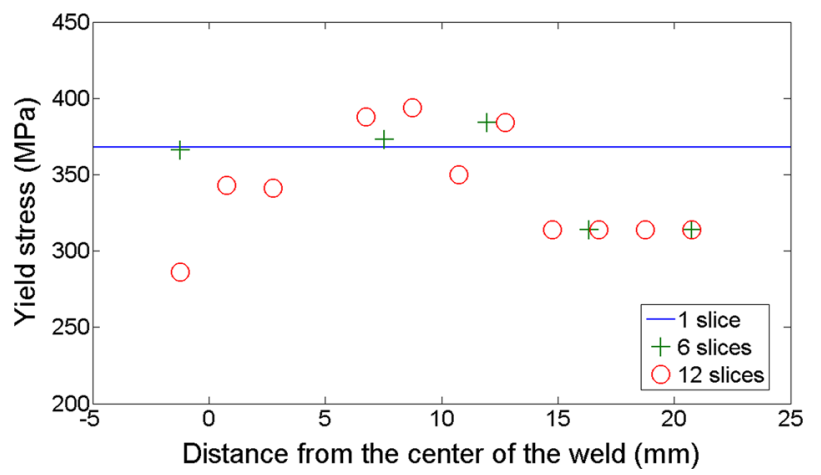

Fig. 13 Evolution of the identified yield stress for a base material specimen depending on the number of slices. The $\mathrm{X}$ axis correspond to the distance from the center of the weld on welded specimen to enable comparison between welded and homogeneous specimens (31 $\times 31$ smoothing window)

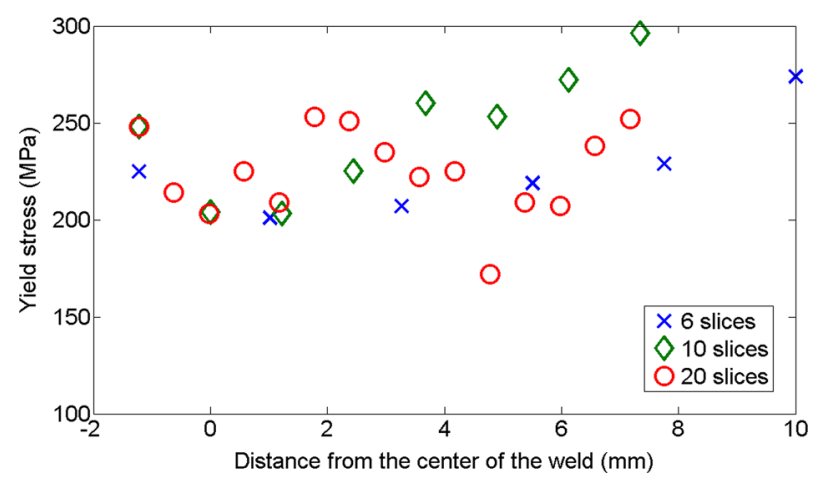

Fig. 14 Influence of the number of slices over the identification of the yield stress for a welded specimen (Grid Method, $31 \times 31$ smoothing window)

not, as shown on Fig. 13. The first thing that is apparent on this figure is that a consistent $20 \%$ reduction of the identified yield stress can be observed on the right-hand side of the specimen. This can be attributed to the very low strain levels experienced there (Fig. 4) compared to the welds (Figs. 6 and 8). The other conclusion that can be drawn is that the number of slices has to be kept low. Looking at the 0 to $15 \mathrm{~mm}$ zone to the left of the graph where strains are large enough for satisfactory identification, one can see that thin slices (12 slices) produce noisy yield stresses, ranging from 280 to $400 \mathrm{MPa}$ whereas thicker slices (6 slices) produce yield stress values that are much less scattered, as one would expect.

The same type of evaluation has been performed on a welded specimen (Fig. 14). As seen in Figs. 6 and 8, the strain levels are larger than for the base material so the identification can be carried out over the whole field of view. It can clearly be seen that an increased slice thickness (i.e. decreased number of slices) leads to a smoother spatial variation of the yield stress. However, when the number of 


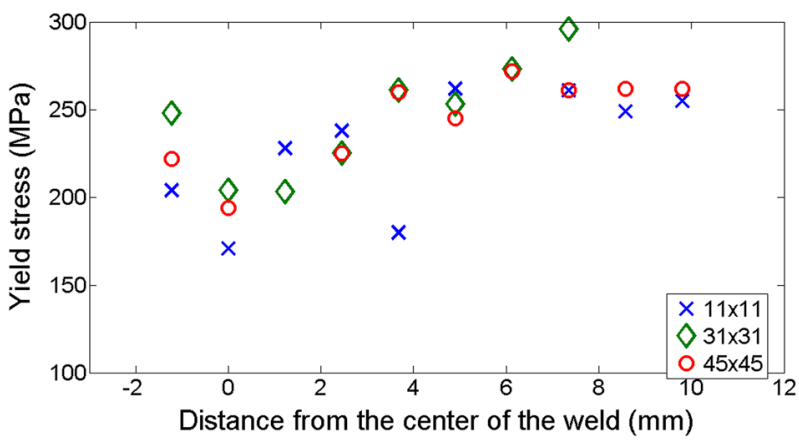

Fig. 15 Influence of the size of the smoothing window (in data points) over the identification of the yield stress for a welded specimen (Grid Method, ten slices)

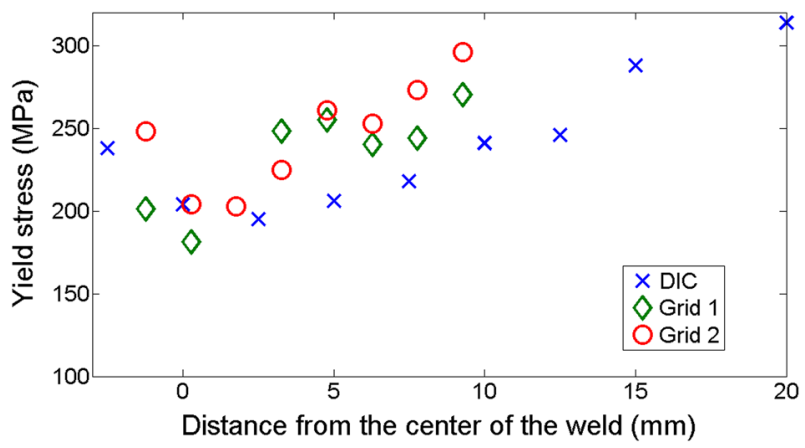

Fig. 16 Identified yield stress for the three dynamic tests on welded specimens (ten slices and $31 \times 31$ smoothing window)

slices goes over 12, it is not possible to identify the yield stress on the second half of the specimen where there is no convergence of the minimisation process anymore. The reason for this is that when the stress state is too uniform spatially, and accelerations are low, then both terms in Eq. 16 are close to zero and convergence cannot be reached (the $\left(2 x_{2}-L\right)$ term has a zero mean over the slice where $x_{2}$ varies between 0 and $L$ ). Increasing the slice width results in higher stress heterogeneity and convergence can be restored. This problem is not evidenced in the welded area where significant strain and stress heterogeneities are present because of the strain localization process. As a result of this compromise, the number of slices will be kept around 9 (and varies slightly for one specimen to the next as field of views and impact speeds are slightly different).

Additionally, the size of the smoothing window can also influence the identified parameters. As stated earlier in this chapter, a $31 \times 31$ window was used for all the tests. Nevertheless, information is lost in the smoothing process, especially when dealing with gradient of properties. The influence of the smoothing on the identification of the yield stress is shown in Fig. 15. As expected, it shows that a larger smoothing window reduces the dispersion of the

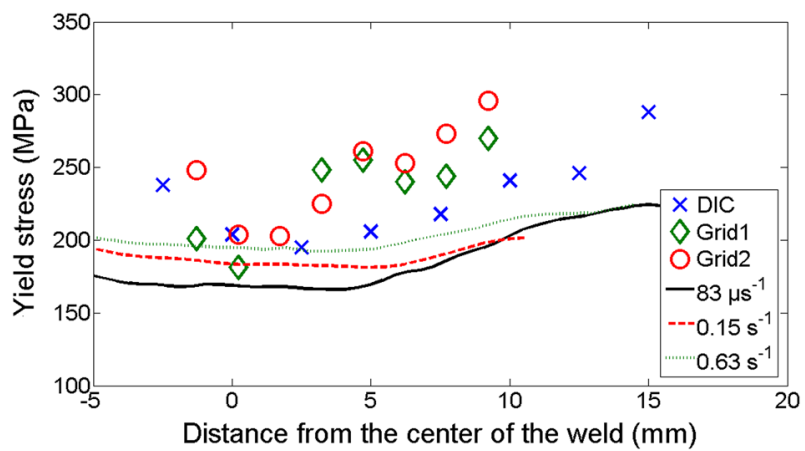

Fig. 17 Identified yield stress for the three dynamic tests (dots) and three quasi-static tests [53] (lines) on welded specimens

results. However, the spatial resolution drops with a larger window size, and it will hinder the measurement of the gradients in mechanical properties. The effect of increased smoothing window is basically the same as that of decreased number of slices.

The final results for the identified yield stress for welded specimens is presented in Fig. 16. It can be seen that very similar identification results have been obtained in the three tests, with evolution of the yield stress throughout the weld exhibiting the lowest value around the center of the weld. The DIC results however provide a much smoother variation as expected because of the reduced spatial resolution of the measurements arising from the very large subset used.

Finally, the current results have been collated with that from [53] to obtain an overview of the variations of yield stress profiles over a large range of strain-rates (Fig. 17). It is interesting to note that at the center of the weld, the identified values show a significant strain-rate sensitivity between $83 \mu \mathrm{s}^{-1}$ and $0.63 \mathrm{~s}^{-1}$, whereas for the base material, sensitivity is towards the larger strain-rates [53]. This is interesting but would need to be backed up with materials science studies to confirm if such an effect is expected. It is clear however that the very different micro-structures between the nugget, heat affected zones and base material could potentially lead to such differences. It must be emphasized that this kind of results would be extremely difficult to obtain by any current method and as such, the present methodology has great future potential to explore local strain-rate sensitivity in welds. This in turn can lead to the development of better visco-plastic constitutive models for such welds. Finally, more complex tests such as three or four point impact bending tests (as in [32]) could be used to identify elastovisco-plastic models over a wider range of stress multiaxiality, which is currently another main limitation of the standard SHPB analysis. 


\section{Conclusion}

A new method for the identification of the dynamic properties of welds has been proposed in this study. It offers a significant contribution to the field of high strain-rate testing. In this work, the acceleration fields have been used as a load cell, in order to carry out the identification of the mechanical properties of the material. While previous work in this area $[23,30]$ was limited to the characterisation of the elastic properties, the identification of both elastic and plastic parameters has been carried out during this study. According to the authors knowledge, it is the first time that the identification of the dynamic yield stress of a material has been attempted without any external load measurement. Moreover, a local characterisation of the dynamic yield stress was performed on welded specimens. The repeatability of the process has been verified on two different set-ups and with two different full-field measurement techniques. The hardware, and more specifically, the high noise level of the cameras and the low number of available images currently remains the main weak point of the method. Moreover, it is essential that in the future, detailed uncertainty assessment of the identified data is performed so that error bars can be added to the yield stress evolutions in Fig. 17. This is a challenging task as the measurement and identification chain is long and complex with many parameters to set. This can only be addressed by using a realistic simulator as developed in [55] for the grid method and more recently [56] for Digital Image Correlation. This enables first to optimize the test and processing parameters (load configuration, subset and smoothing in DIC, virtual fields in the VFM) and then to provide uncertainty intervals for the identified parameters. This has recently been validated experimentally in [57]. The present case will be more computationally challenging but conceptually, the procedures in [55-57] can be used in exactly the same way as for elasticity. This will have to be investigated in the near future when tests with better images are available. It is a key issue for making this new procedure a standard technique for which users have confidence in the results.

It is believed that the demonstrated ability to extract local material properties without the requirement for external load measurement will open unprecedented opportunities to expand the range of experimental approaches that can be used in the field of high strain-rate testing. To develop the next generation of novel methodologies and make them available to researchers and engineers, significant additional research will be required, with the growth and continuous improvement of modern high speed imaging technology being the foundation for the effort.

Acknowledgments The authors would like to thank the Champagne-Ardenne Regional Council for funding $50 \%$ of the Ph.D. studentship of G. Le Louëdec. The authors would also like to acknowledge the support of the US Army Research Office through ARO Grants $\sharp$ W911NF-06-1-0216 and Z-849901, and the NSF through the I/UCRC Center for Friction Stir Processing.

\section{References}

1. Thomas WM, Nicholas ED, Needham JC, Murch MG, Templesmith P, Dawes CJ (1991) Friction-stir butt welding, GB patent No. 9125978.8, international patent application No. PCT/GB92/ 02203

2. Field J, Walley S, Proud W, Goldrein H, Siviour C (2004) Review of experimental techniques for high rate deformation and shock studies. Int J Impact Eng 30(7):725-775

3. Hopkinson B (1914) A method of measuring the pressure produced in the detonation of high explosives or by the impact of bullets. Phil Trans R Soc 213(10):437-452

4. Kolsky H (1949) An investigation of the mechanical properties of materials at very high rates of loading. Proc Phys Soc Sect B 62(11):676-700

5. Harding J, Wood E, Campbell J (1960) Tensile testing of materials at impact rates of strain. J Mech Eng Sci 2:88-96

6. Jiang C, Chen M (1974) Dynamic properties of materials, part II: Aluminum alloys. Defense Technical Information Center

7. Nicholas T (1981) Tensile testing of materials at high rates of strain - an experimental technique is developed for testing materials at strain rates up to $103 \mathrm{~s}^{-1}$ in tension using a modification of the split Hopkinson bar or Kolsky apparatus. Exp Mech 21:177-185

8. Jenq S, Sheu S (1994) An experimental and numerical analysis for high strain rate compressional behavior of 6061-O aluminum alloy. Comput Struct 52:27-34

9. Smerd R, Winkler S, Salisbury C, Worswick M, Lloyd D, Finn M (2005) High strain rate tensile testing of automotive aluminum alloy sheet. Int J Impact Eng 32:541-560

10. Zhang X, Li H, Li H, Gao H, Gao Z, Liu Y, Liu B (2008) Dynamic property evaluation of aluminum alloy 2519A by split hopkinson pressure bar. Trans Nonferr Met Soc China 18:1-5

11. Hadianfard M, Smerd R, Winkler S, Worswick M (2008) Effects of strain rate on mechanical properties and failure mechanism of structural Al-Mg alloys. Mater Sci Eng A 492:283-292

12. Abotula S, Chalivendra V (2010) An experimental and numerical investigation of the static and dynamic constitutive behaviour of aluminium alloys. J Strain Anal Eng Des 45:555-565

13. Tucker M, Horstemeyer M, Whittington W, Solanki K, Gullett $P$ (2010) The effect of varying strain rates and stress states on the plasticity, damage, and fracture of aluminum alloys. Mech Mater 42:895-907

14. Chen W, Song B (2009) Dynamic characterization of soft materials. In: Shukla A, Ravichandran G, Rajapakse YD (eds) Dynamic failure of materials and structures. Springer, New York, pp 1-28

15. Hoge K (1966) Influence of strain rate on mechanical properties of 6061-T6 aluminum under uniaxial and biaxial states of stress. Exp Mech 6:204-211

16. Xu Z, Li Y (2009) Dynamic behaviors of 0Cr18Ni10Ti stainless steel welded joints at elevated temperatures and high strain rates. Mech Mater 41(2):121-130

17. Lee W-S, Lin C-F, Liu C-Y, Tzeng F-T (2004) Impact properties of 304L stainless steel GTAW joints evaluated by high strain rate of compression tests. J Nucl Mater 335(3):335-344

18. Zhang J, Tan C, Ren Y, Wang F, Cai H (2011) Quasi-static and dynamic tensile behaviors in electron beam welded Ti-6Al-4V alloy. Trans Nonferr Met Soc China 21(1):39-44

19. Yokoyama T, Nakai K, Kotake Y (2007) High strain rate compressive stress strain response of friction stir welded 7075-T651 
aluminum alloy joints in through thickness direction. J Jpn Inst Lights Met 57:518-523

20. Reu P, Miller T (2008) The application of high-speed digital image correlation. J Strain Anal Eng Des 43(8):673-688

21. Pierron F, Cheriguene R, Forquin P, Moulart R, Rossi M, Sutton M (2011) Performances and limitations of three ultra high-speed imaging cameras for full-field deformation measurements. In: Series: advances in experimental mechanics VIII, applied mechanics and materials (Trans Tech Publications), vol 70. Joint BSSM/SEM ISEV conference, 7-9 in Edinburgh (UK)

22. Tiwari V, Sutton M, McNeill S (2007) Assessment of high speed imaging systems for 2D and 3D deformation measurements: methodology development and validation. Exp Mech 47:561-579

23. Pierron F, Zhu H, Siviour C (2014) Beyond Hopkinson's bar. Philos Trans R Soc A 372:20130195

24. Pierron F, Grédiac M (2012) The virtual fields method. Springer, New-York

25. Avril S, Pierron F (2007) General framework for the identification of constitutive parameters from full-field measurements in linear elasticity. Int J Solids Struct 44(14-15):4978-5002

26. Pannier Y, Avril S, Rotinat R, Pierron F (2006) Identification of elasto-plastic constitutive parameters from statically undetermined tests using the virtual fields method. Exp Mech 46(6):735-755

27. Grédiac M, Pierron F (2006) Applying the virtual fields method to the identification of elasto-plastic constitutive parameters. Int J Plast 22:602-607

28. Avril S, Pierron F, Sutton MA, Yan J (2008) Identification of elasto-visco-plastic parameters and characterization of Lüders behavior using digital image correlation and the virtual fields method. Mech Mater 40(9):729-742

29. Sutton MA, Yan JH, Avril S, Pierron F, Adeeb SM (2008) Identification of heterogeneous constitutive parameters in a welded specimen: uniform stress and virtual fields methods for material property estimation. Exp Mech 48(4):451-464

30. Moulart R, Pierron F, Hallett S, Wisnom M (2011) Full-field strain measurement and identification of composites moduli at high strain rate with the virtual fields method. Exp Mech 51:509-536

31. Pierron F, Forquin P (2012) Ultra high speed full-field deformation measurements on concrete spalling specimens and stiffness identification with the virtual fields method. Strain 28(5): 388-4058

32. Pierron F, Sutton M, Tiwari V (2011) Ultra high speed DIC and virtual fields method analysis of a three point bending impact test on an aluminium bar. Exp Mech 51(4):537-563

33. Huntley J (1998) Automated fringe pattern analysis in experimental mechanics: a review. J Strain Anal Eng Des 33: 105-125

34. Surrel Y (2000) Fringe analysis. Photomechanics, vol 77 of topics in applied physics, pp 55-102

35. Surrel Y (1997) Design of phase-detection algorithms insensitive to bias modulation. Appl Opt 36:805-807

36. Badulescu C, Grédiac M, Mathias J, Roux D (2009) A procedure for accurate one-dimensional strain measurement using the grid method. Exp Mech 49(6):841-854

37. Badulescu C, Grédiac M, Mathias J (2009) Investigation of the grid method for accurate in-plane strain measurement. Meas Sci Technol 20(9):095102
38. Ri S, Fujikagi M, Morimoto Y (2010) Sampling moiré method for accurate small deformation distribution measurement. Exp Mech 50(4):501-508

39. Ri S, Muramatsu T, Saka M, Nanbara K, Kobayashi D (2012) Accuracy of the sampling moiré method and its application to deflection measurements of large-scale structures. Exp Mech 52(4):331-340

40. Sutton MA, Orteu J, Schreier HW (2009) Image correlation for shape., Motion and deformation measurementsSpringer, NewYork

41. Piro J-L, Grédiac M (2006) Producing and transferring low-spatial-frequency grids for measuring displacement fields with moiré and grid methods. Exp Tech 28:23-26

42. Surrel Y (1993) Phase stepping: a new self-calibrating algorithm. Appl Opt 32:3598-3600

43. Surrel Y (1996) Design of algorithms for phase measurements by the use of phase stepping. Appl Opt 35:51-60

44. Ghiglia DC, Mark PD (1998) Two dimensional phase unwrapping: theory, algorithms \& software. Wiley-Interscience publication, New York

45. Baldi A, Bertolino F, Ginesu F (2002) On the performance of some unwrapping algorithms. Opt Lasers Eng 37:313-330

46. Zappa E, Busca G (2008) Comparison of eight unwrapping algorithms applied to fourier-transform profilometry. Opt Lasers Eng 46:106-116

47. Bioucas-dias J, Valadao G (2007) Phase unwrapping via graph cuts. IEEE Trans Image Process 16:698-709

48. Gorry PA (1990) General least-squares smoothing and differentiation by the convolution (Savitzky-Golay) method. Anal Chem 62(6):570-573

49. VIC2D, Correlated Solutions Incorporated, 120 Kaminer Way, Parkway Suite A, Columbia SC 29210, www.correlatedsolutions. com

50. Sutton MA, Deng X, Liu J, Yang L (1996) Determination of elastic-plastic stresses and strains from measured surface strain data. Exp Mech 36(2):99-112

51. Olsson D, Nelson L (1975) Nelder-mead simplex procedure for function minimization. Technometrics 17:45-51

52. Pierron F, Avril S, Tran V (2010) Extension of the virtual fields method to elasto-plastic material identification with cyclic loads and kinematic hardening. Int J Solids Struct 47:2993-3010

53. Le Louëdec G, Pierron F, Sutton MA, Reynolds AP (2013) Identification of the local elasto-plastic behavior of FSW welds using the virtual fields method. Exp Mech 53(5):849-859

54. Reemsnyder H, Throop J (1982) Residual stress effects in fatigueSTP 776. American Society for Testing \& Materials

55. Rossi M, Pierron F (2012) On the use of simulated experiments in designing tests for material characterization from full-field measurements. Int J Solids Struct 49(3-4):420-435

56. Rossi M, Lava P, Pierron F, Debruyne D, Sasso M (2015) Effect of DIC spatial resolution, noise and interpolation error on identification results with the $\mathrm{vfm}$. Strain, in revision

57. Wang P, Pierron F, Thomsen O, Rossi M, Lava P (2015) Uncertainty quantification in VFM identification, vol 6. Springer, New York, pp 137-142 